\title{
Human papillomavirus detection by hybrid capture and its possible clinical use
}

\author{
A Farthing, P Masterson, W P Mason, K H Vousden
}

\begin{abstract}
Aims-To determine the sensitivity of the hybrid capture method for human papillomavirus (HPV) detection and potential clinical uses as a screening method for the identification of cervical intraepithelial neoplasia.

Methods-The presence of oncogenic types of HPV was tested for in samples taken from the cervix at colposcopy, and compared with detection by polymerase chain reaction (PCR) in 60 patients. Both sets of results were corrected with the pathology determined by biopsy and smear cytology.

Results-Hybrid capture detection showed $86 \%$ agreement with PCR. Eighty three percent of CIN 3 lesions, $62 \%$ of CIN 2, 59\% of CIN 1 and $21 \%$ of normal controls were positive for oncogenic HPV types.

Conclusion-The hybrid capture detection method is reliable, sensitive, and easy to use. The addition of HPV testing to cytological screening would detect a greater proportion of cervical dysplasia with a higher false positive rate.
\end{abstract}

$(\mathcal{C}$ Clin Pathol 1994;47:649-652)

The association between cervical cancer and the presence of human papillomavirus (HPV) has been well established. ${ }^{2}$ About $90 \%$ of cervical malignancies contain HPV compared with $10 \%$ of the normal population. ${ }^{3}$ There are in excess of 60 different HPV types only some of which are associated with genital lesions. Types $6,11,42,43$ and 44 occur in benign genital warts; types 16, 18, 31, 33 and others are found in cervical cancers. ${ }^{4}$ The latter "high risk" or oncogenic group can also immortalise human epithelial keratinocytes in culture and cause them to behave like cells from cervical intraepithelial neoplasia (CIN). ${ }^{5}$ Untreated CIN 3 progresses to invasive cancer in $36 \%$ of cases over 20 years, ${ }^{6}$ and these lesions frequently contain HPV. In a previous representative series $77 \%$ of $\mathrm{CIN} 2$ or 3 lesions contained high risk HPV types. ${ }^{3}$

Certain HPV types have a strong association with, and probably a causative role in, the pathogenesis of premalignant cervical lesions. Therefore, detection of this virus could help to identify early and eradicate $\mathrm{CIN}$, thereby decreasing the rate of invasive cancer. A recent study set in a sexually transmitted dis- ease clinic identified a group of women who had no cytological cervical abnormality but were HPV positive. ${ }^{7}$ Within two years $28 \%$ had developed CIN 2 or 3 compared with $3 \%$ of controls. The highest risk was associated with HPV 16. Screening by cervical cytology alone has limitations with a false negative rate estimated at nearly $20 \% .^{8}$ Mild dyskaryosis can also conceal an underlying lesion of CIN 2 or 3 in up to $50 \%{ }^{9}$ and Cuzick et al ${ }^{10}$ have suggested that the presence of intermediate or high amounts of HPV 16 are associated with these lesions. There could be advantages if cytology were replaced with or supplemented by HPV testing.

Because serological analyses are not available, HPV is detected by identifying the presence of its DNA in a specimen. Histological features suggestive of HPV are an extremly poor predictor of the presence of DNA. Various techniques have been established which are more sensitive and reliable methods of HPV detection. In situ hybridisation involves the probing of histological samples and visualising the stained nuclei which contain HPV under microscopic vision. This is relatively inaccurate and has been superseded by molecular biological methods. Southern hybridisation detects HPV down to about 5000 copies per sample and is still regarded as the standard reference method. ${ }^{11}$ However, its widespread application is limited because it is time consuming, can be technically difficult, and often relies on the use of ${ }^{32} \mathrm{P}$ radiolabelled probes and the subjectivity of autoradiographic interpretation. Virapap and viratype dot blot methods have proved accurate but also rely on radioactive isotopes. The polymerase chain reaction (PCR) is the most sensitive method involving amplification of the DNA before detection either on an agarose gel or Southern blot. This will detect as little as 10 copies per sample. However, the risks of amplifying contaminating HPV DNA and obtaining false positive results are substantial. Although many current studies have used PCR, this drawback could preclude the use of the technique on any large scale for clinical purposes. The hybrid capture viratype test (Digene Diagnostics, Silver Spring, Maryland, USA) has been developed as a simple non-radioactive method that is sensitive for detecting HPV. Agreement between this method and Southern hybridisation is $90 \%$ (Impraim C, abstract presented at 92nd General meeting of the American Society of 
Microbiology, 1992). The purpose of this study was to evaluate the test for possible further widespread use.

\section{Methods}

Two hundreds colposcopic examinations were performed on 162 consecutive patients by one clinician (AF). The cohort was a mix of 73 new referrals, 27 patients undergoing treatment by large loop excision of the transformation zone, and 100 being followed up, including those treated before. At the beginning of each examination a cytological smear, including an endocervical brush, was taken under direct colposcopic vision and sent for routine testing. The "viratype" swab was used to collect desquamated cells from the ectocervix and transformation zone and placed in transport medium. This was stored at $-20^{\circ} \mathrm{C}$ until analysis. A routine colposcopic examination was then performed taking directed biopsy specimens from areas of abnormality. These were sent for routine histological examination.

In 60 unselected consecutive patients samples were also obtained for the detection of HPV by PCR. After the routine cytological smear had been taken the spatula was passed over the cervix again and the cells collected by shaking them into phosphate buffered saline.

A detailed methodology for the hybrid capture technique has already been described (Impraim C, abstract presented at General Meeting of the American Society of Microbiology, 1992). In this study we only used the kit probe which detects the oncogenic types of HPV. It recognises a consensus region of types $16,18,31,33,35,45,51,52$ and 56. Briefly, the DNA from the lysed cells is denatured and an RNA probe added which binds to the single stranded HPV DNA. This hybrid is then captured by an antibody coating the inside of a tube. Addition of an alkaline phosphatase conjugate causes a chemoluminescent reaction, the intensity of which can be measured. A result was regarded as positive if the numerical chemoluminescent

Table 1 Correlation between hybrid capture and PCR

\begin{tabular}{llcl}
\hline Hybrid capture & $P C R$ positive & $P C R$ negative & Total \\
\hline Positive & 12 & 5 & 17 \\
Negative & 3 & 35 & 38 \\
Total & 15 & 40 & 55 \\
\hline
\end{tabular}

Sensitivity $17 / 20$ (85\%); specificity $35 / 40(87 \%)$; agreement $47 / 55(86 \%)$.

Table 2 Correlation between HPV status, cytology and biopsy results

\begin{tabular}{llcccc}
\hline \multirow{2}{*}{$\begin{array}{l}\text { Biopsy } \\
\text { result }\end{array}$} & HPV & \multicolumn{2}{l}{ Cytology } & \\
\cline { 2 - 4 } & positive & Action & Recall & Negative & Cytology $+H P V$ \\
\hline CIN 3 & $20 / 24(83 \%)$ & $24 / 24$ & $0 / 24$ & $0 / 24$ & $24 / 24(100 \%)$ \\
CIN 2 & $10 / 16(62 \%)$ & $7 / 16$ & $3 / 16$ & $6 / 16$ & $11 / 16(69 \%)$ \\
CIN 1 & $10 / 17(59 \%)$ & $5 / 17$ & $6 / 17$ & $6 / 17$ & $11 / 17(65 \%)$ \\
Normal & $12 / 38(32 \%)$ & $1 / 38$ & $11 / 38$ & $25 / 38$ & $22 / 107(21 \%)$ \\
No biopsy & $9 / 69(16 \%)$ & $2 / 69$ & $3 / 69$ & $66 / 73$ & \\
\hline Cytology is divided into Action (severe or moderate dyskaryosis), Recall (mild or borderline \\
dyskaryosis), or Negative (normal smear). No biopsy specimen was taken if the colposcopy was \\
normal.
\end{tabular}

reading from the sample was greater than one multiple of the positive control.

The material and methods used in the PCR reactions have been described elsewhere. ${ }^{12}$ Consensus primers which recognise the L1 region of HPV types $6,11,16,18,31,33,42$, 45 and other uncharacterised HPV types were used in a $100 \mu \mathrm{l}$ PCR reaction for 35 cycles at $92^{\circ} \mathrm{C}$ for one minute, $55^{\circ} \mathrm{C}$ for one minute, and $72^{\circ} \mathrm{C}$ for one minute. Primers amplifying a 256 base pair fragment of the $\beta$ globin gene were also included in the reaction as an internal standard. A 1 in 10 dilution of the reaction was run on a $1 \%$ agarose gel and DNA detected under ultraviolet light. All samples where DNA has been successfully extracted should have the $\beta$ globin band and those positive for HPV an additional one at 450 base pairs. The DNA was transferred to a nitrocellulose membrane using Southern blotting and specific HPV types detected with biotinylated probes using the enhanced chemoluminescence (ECL) detection system. Each membrane was used in separate reactions to detect types 6 or 11,16 alone, 18 alone, and 31,33, or 35.

HPV detection rates by hybrid capture were compared with the histological and cytological results and the PCR method. All comparisons were performed on samples taken at the same visit.

\section{Results}

The comparison of hybrid capture and PCR is shown in table 1. $\beta$ globin was amplified in 55 of the 60 PCR samples. There was overall agreement on the HPV reaction in 47 of 55 $(86 \%)$. The hybrid capture detected more positive samples (17 to 15$)$ in this study and was negative in only three cases where PCR was positive.

Having shown that there was good correlation between these two methods of HPV detection, the results of the hybrid capture system were further analysed with regard to the cervical pathology. HPV testing, cytology, and, where indicated, colposcopic directed biopsy were performed on each patient. The biopsy result was used as the reference for comparison of the two screening methods shown in table 2 . An HPV reaction is positive if the chemoluminescence is greater than one multiple of the positive control. The smear reports are divided according to present policy in our region. Action is required if moderate or severe dyskaryosis is present; mild dyskaryosis is labelled recall and a normal smear as negative. Samples reported showing histological atypia, dysplasia of unknown grade, or patients who were either pregnant or returning for treatment of a lesion already analysed were excluded from this table, leaving 169 samples.

The oncogenic types of HPV were detected in $83 \%$ of CIN 3, $62 \%$ of CIN 2, and $59 \%$ of CIN 1 lesions. In those patients with a normal biopsy specimen HPV was detected only in $32 \%$. A biopsy specimen was not taken if the cervix was colposcopically normal. 
All CIN 3 lesions in this group would have been detected by positive cytology. However, this applies to only seven of $16(44 \%)$ of $\mathrm{CIN}$ 2 lesions. In fact six of 17 (35\%) of these had a normal smear and would have been reassured that further cytological examination was not necessary for three years if they had been screened in the community. Similar figures apply to the detection of CIN 1 by smear.

If HPV detection was to be used as a screening test instead of cytology in this series it would only have detected $83 \%$ of CIN 3 and $62 \%$ of $\mathrm{CIN} 2$ lesions while regarding as abnormal $21 \%$ of women with a normal cervix (determined by biopsy or colposcopy).

This assay is quantitative and the values obtained varied between $1 \cdot 1$ and 212 multiples of the positive control. There was no correlation in this study between the amount of HPV detected and the underlying pathology. CIN 3 mean 29.6 (SD 45), CIN 2 mean 23.1 (SD 35), CIN 1 mean 40.9 (SD 58) and normal controls mean 20.6 (SD 26).

Interestingly, of eight women who had HPV before treatment and returned for follow up during the trial, seven $(87 \%)$ were subsequently HPV negative. The only patient who remained positive had residual disease in the endocervix.

Of 22 patients with a smear reported as mild dyskaryosis, 13 (59\%) were positive for HPV, with four showing a high grade underlying lesion, three $(75 \%)$ of which were positive for HPV DNA by hybrid capture.

\section{Discussion}

The results of this study confirm that the hybrid capture assay detects oncogenic types of HPV in most dysplastic cervical lesions. The higher the grade of histological abnormality, the more likely it is to contain HPV. The assay seems to be at least as sensitive as PCR in the sample population investigated. The entire procedure takes six hours of which four are needed for either incubation or hybridisation steps. It is a simple technique which could be readily automated for large scale use if required. As there is no amplification of DNA there is little risk of contamination giving a false positive result. The chemoluminescence reading is set against a standard positive control solution and the digital readout is then quantitative removing the inaccuracy of subjective interpretation. One drawback, however, is the lack of an internal standard which indicates an adequate sample has been obtained. This was highlighted in our study because, of the four patients with CIN 3 who had no HPV detected on their initial visit, two were positive when they returned for treatment. This suggests that insufficient cells from the appropriate part of the cervix were obtained the first time. It may also explain the cases which were positive by PCR but negative by hybrid capture. Some cases were surprisingly positive by the hybrid capture method but negative by PCR. These were possibly due to the lack of conservation of part of the L1 region of the HPV genome which may occur in HPV positive cervical carcinoma.

The hybrid capture kit also allows nononcogenic types of HPV to be detected with a probe which identifies types $6,11,42,43$ and 44 but as these types are less frequently associated with cervical dysplasia we omitted to test for them.

This cohort of women was being referred, or had been treated for cervical abnormality, and therefore are not fully representative of the general population. However, certain conclusions on the merits of cytology with HPV testing as a screening procedure can be drawn which are consistent with previous reports. ${ }^{13} \mathrm{~A}$ combination of the two tests detected a greater proportion of high grade dysplasia than either method alone, particularly for CIN 2 which was poorly detected by cytology. This improvement is weighed against the large number of false positive results which, if referred for colposcopy, would further overload an already burdened system. However, as most false positive results are due to HPV infection, reducing the number of HPV types the test detects will probably increase specificity. Further work is required to determine the best combination for good sensitivity and specificity.

The observation that all the HPV positive women adequately treated were subsequently HPV negative carries implications for future follow up. Cytology without colposcopy has been proved to be inadequate in the immediate follow up of treated patients. ${ }^{14}$ Any reliable alternative to colposcopy would, however, allow resources to be concentrated on appropriately referred new patients. More extensive study is required to determine whether HPV testing with or without cytology will fulfil the criteria.

Although numbers were small, this study confirms that high grade cervical lesions reported as showing mild dyskaryosis are more likely to be positive for oncogenic types of HPV.

The ultimate aim of cervical screening is to prevent cervical cancer and further studies are required to determine whether the addition of HPV testing will achieve this. Some types of HPV, such as HPV 31, are found relatively frequently in CIN 3 but are rarely seen in cancer. This may in part explain why not all CIN 3 progresses to invasive disease. But HPV typing may help to determine which $\mathrm{CIN}$ lesions pose the greatest risk. With an increased risk of premature delivery shown even after laser conisation, ${ }^{15}$ more specific identification of the lesions requiring treatment is highly desirable.

For HPV detection hybrid capture is easier and more suited to widespread use than PCR, but the benefits of this as a screening method with increased CIN detection are at present outweighed by the large number of false positive results for underlying pathology.

\footnotetext{
1 Zur Hausen. HPV in pathogenesis of anogenital cancer Virology 1991;184:9-13.

2 Crook T, Farthing A. Human papillomavirus and cervical
} 
cancer. Br $\mathcal{F}$ Hosp Med 1993;49:131-2.

3 Lorincz A, Reid R, Jenson AB, Greenberg MD, Lancaster W, Kurman RJ. Human papillomavirus infection of the cervix: relative risk associations of fifteen common anogenital types. Obstet Gynaecol 1992; 79:328-37.

4 Lorincz A, Temple GF, Kurman RJ, Jenson AB, Lancaster WB. Oncogenic association of specific human papillomavirus types with cervical neoplasia. $尹 N C I$ 1987; mavirus $671-7$.

5 McCance D, Kopan R, Fuchs E, Laimens LA. Human papillomavirus type 16 alters human epithelial cell differentiation in vitro. Proc Natl Acad Sci USA 1988, 85:7169-73.

6 McIndoe WA, McLean MR, Jones RW, Mullins PR. The invasive potential of carcinoma in situ of the cervix. Obstet Gynaecol 1984;64:451-8.

7 Koutsky LA, Holmes KK, Critchlow CW, Stevens CE, Paavonen J, Beckmann AM, et al. A cohort study of the relative risk of cervical intraepithelial neoplasia grade 2 or 3 in relation to papillomavirus infection. $N$ Engl $\mathcal{F} M e d$ 1992;327:1272-8.

8 Beilby JOW, Bourne R, Guilleband J, Steele ST. Paired cervical smears, a method of reducing the false negative rate in a screening population. Obstet Gynaecol 1982, 60:46-8.
9 Soutter WP, Wisdom S, Brough AK, Monaghan JM Should patients with mild atypia in a cervical smear be
referred for colposcopy? $\mathrm{Br} f$ Obstet Gynaecol 1986;93:70-4.

10 Cuzick J, Terry G, Ho L, Hollingworth T, Anderson M Human papillomavirus type 16 DNA in cervical smears as predictor of high grade cervical neoplasia. Lancet as predictor of high

11 Schneider A. Pathogenesis of genital HPV infection. Genitourin Med 1993;69:165-73.

12 Bauer HM, Ting Y, Greer C, Chambers J, Tashiro CJ, Chimera J, et al. Genital human papillomavirus infection in female university students as determined by a PCR based method $\mathcal{F} A M A 1991 ; 265: 472-7$.

13 Reid R, Greenberg MD, Lorincz AT, Jenson AB, Laverty $C R$, Husain $M$. Should cervical cytologic testing be augmented by cervicography or human papillomavirus deoxyribonuoleic acid detection? Am $\mathcal{F}$ Obstet Gynaecol 1991;164:1461-71.

14 Mahadevan N, Horwell D. The value of colposcopy in the follow up of cervical intraepithelial neoplasia after treatment by laser excision. Br $\mathcal{F}$ Obstet Gynaecol 1993; ment by $563-6$.

15 Hagen B, Skeldestad F. The outcome of pregnancy after $\mathrm{CO}_{2}$ laser conisation of the cervix. Brf Obstet Gynaecol 1993;100:717-20. 